Pacific Journal of Mathematics

ON LIAPUNOV FUNCTIONS WITH A SINGLE CRITICAL 


\title{
ON LIAPUNOV FUNCTIONS WITH A SINGLE CRITICAL POINT
}

\author{
WALTER LEIGHTON
}

In this paper we discuss the geometry of the level surfaces of functions $f(x)=f\left(x_{1}, x_{2}, \cdots, x_{n}\right)$ of class $C^{\prime \prime}$ in $E^{n}$ that possess an isolated relative minimum point at the origin, and no other critical points, finite or infinite. Our principal result is that such a function satisfies the condition $f(x)>f(0)$ for all $(x) \neq(0)$. The levels sets $f(x)=c$ and the domains they bound are discussed. The results are useful in Liapunov stability theory.

A finite critical point of $f(x)$ is a point of $E^{n}$ at which $f_{x_{i}}=0$ $(\imath=1,2, \cdots, n)$. We shall say that $f(x)$ possesses an infinite critical point if there is some sequence of point $\left\{x_{n}\right\},\left(x^{n}\right) \rightarrow \infty$, for which the function

$$
F(x)=f_{x_{1}}^{2}+f_{x_{2}}^{2}+\cdots+f_{x_{n}}^{2}
$$

tends to zero. To say that $f(x)$ has no infinite critical point means then that there exists a positive constant $\varepsilon$ and a sphere $\|x\|=r^{2}$ such that $F(x) \geqq \varepsilon$ outside the sphere.

Let $f(x)=f\left(x_{1}, x_{2}, \cdots, x_{n}\right)$ be a function of class $C^{\prime \prime}$ in $E^{n}$, and suppose that $f(x)$ is positive definite neighboring the origin; that is $f(0)=0$, and $f(x)>0$ near the origin. We term such a function $f(x)$ admissible. The point $x=0$ is then a relative minimum point (possibly degenerate) of $f(x)$. Suppose that $f(x)$ is admissible and that it has no critical point, finite or infinite, except at the origin. It follows that $F(x)$ is bounded away from zero in the complement of every spherical ball with center at the origin. There is then a sphere $S$ with center at 0 on which $f>0$. Let $m$ be the minimum value assumed by $f$ on $S$, and consider the set of points $M$ inside $S$ for which $f=a(0<a<m)$. This set clearly exists, for consider the continuous function $F$ along any continuous arc joining the origin to a point on $S$ where $f=m$. $M$ has the following properties. At each point of $M$ the implicit-function theorem guarantees that the equation

$$
f(x)=a
$$

can be solved for one of the variables $x_{i}$, inasmuch as at least one of the functions $f_{x_{i}} \neq 0$ at each point of $M$. This solution will be locally of class $C^{\prime}$ in the remaining variables. It follows that an open neighborhood of each point of $M$ is a homeomorphic image of an open 
disk in $E^{n-1}$ and, consequently, that $M$ is a closed manifold. ${ }^{1}$ Next, we shall show that $M$ bounds a domain containing the origin. Consider any continuous arc joining the origin to an arbitrary point of $S$. At some point of this arc $f$ must assume the value $a$. This point belongs to $M$. It should be observed that $M$ is connected, for if it were not, it would be composed of a set of bounded, complementary domains in each of which $f$ would have a minimum point. This would contradict the assumption of the existence of only one critical point.

The curves orthogonal to the family of level surfaces $f(x)=c$ are solutions of the system [see 2]

$$
\frac{d x_{i}}{d \tau}=\frac{f_{x_{i}}}{f_{x_{1}}^{\prime 2}+f_{x_{2}}^{\prime 2}+\cdots+f_{x_{n}}^{\prime 2}} \quad(i=1,2, \cdots, n) .
$$

Further, Morse [3] shows that writing the differential equations for the orthogonal trajectories in the form (2) permits a parametrization $x=x(\tau)$ of each trajectory with the property

$$
f[x(\tau)] \equiv \tau .
$$

It follows that $f \not \equiv$ constant on any subarc of the trajectory. Because of our assumption of the absence of critical points, except the origin, fundamental existence theorems guarantee that there is a unique solution without multiple points of (2) through each point of $E^{n}-\{0\}$, and that this solution can be extended (in both directions along the curve) to the boundary of $E^{n}-\{0\}$. It follows that each trajectory goes from the origin to infinity as $\tau$ increases steadily from the value zero.

Further, if $\tau$ ranges on a finite interval $0<\tau_{0} \leqq \tau \leqq \tau_{1}$, we shall see that the functions $x_{i}(\tau)$ remain bounded. It will follow (since the trajectories go from the origin to infinity) that $\tau$, and hence $f$, increases steadily from 0 to $+\infty$ along each trajectory. To prove this note that (1) implies that each of the $n$ functions

$$
\frac{f_{x_{i}}}{f_{x_{1}}^{2}+f_{x_{2}}^{2}+\cdots+f_{x_{n}}^{2}}=\frac{f_{x_{i}}}{F}
$$

is bounded outside a sufficiently small sphere $S_{0}$ having the origin as its center. If $M$ denotes a common bound of these quotients, we have from (2) that

$$
x_{i}(\tau)=c_{i}+\int_{\tau_{0}}^{\tau} \frac{f_{x_{i}}[x(\tau)]}{F\lfloor x(\tau)]} d \tau \quad(i=1,2, \cdots, n),
$$

${ }^{1}$ In addition to being locally euclidean $M$ is clearly bounded. Further, since $f$ is continuous, $f^{-1}(a)$, the map of a closed set (single point) of $R$, is also closed, accordingly, $M$ is compact. 
and

$$
\left|x_{i}(\tau)\right| \leqq\left|c_{i}\right|+M\left(\tau-\tau_{0}\right) .
$$

Here, $c_{i}$ is a constant, and $\tau_{0}$ is the value assumed by $f$ at the point where the trajectory pierces $S_{0}$.

The set of points $M: f=a$ has been shown to be a closed bounded manifold. If the set of points $f=c_{0}\left(c_{0}>a\right)$ is a closed bounded manifold bounding an open domain $D$ containing the manifold $M$, and if $D$ contains no critical point except the origin, Morse's program in [3] is readily extended to show that the sets $f \leqq c$ and $f \leqq a\left(a \leqq c \leqq c_{0}\right)$ are homeomorphic. The question arises as to how large $c_{0}$ may be taken in the present analysis. To answer this consider the family of trajeetories orthogonal to $M$ each parametrized so that (3) holds. Let $c_{0}(>a)$ be any value assumed by $f$ and extend each trajectory from $\tau=a$ to $\tau=c_{0}$. Each such endpoint $\tau=c_{0}$ of a trajectory clearly lies on the level surface $f=c_{0}$ We shall show that these "ends" constitute a closed bounded manifold.

To accomplish this, note that we may show, as above, that the functions $x(\tau)$ are bounded for $a \leqq \tau \leqq c_{0}$. Next, let $P$ be any point where $f=c_{0}$. There is a unique solution of (2) through $P$, and it can be parametrized so that (3) holds. Extend that trajectory in the direction of decreasing $\tau$ to $\tau=a$. This point clearly lies on $M$, and the trajectory is the unique trajectory orthogonal to $M$, at this point. Thus, the set of points $f=c_{0}$ are bounded, and the trajectories provide a one-to-one continuous mapping of the set $f \leqq a$ into the set $f \leqq \boldsymbol{c}_{0}$, precisely as in Morse's analysis.

Now let $M_{1}$ be any bounded closed manifold determined by the equation $f(x)=c_{1}$ that bounds a domain $D_{1}$ containing the origin, and let $P$ be any point of $D_{1}$ inside $M_{1}$. We shall show that $f(P)<c_{1}$. For, consider the trajectory $T: x=x(\tau)$ through $P$ orthogonal to $M_{1}$, and suppose that $f(P) \geqq c_{1}$. The function $f[x(\tau)]$ is of class $C^{\prime}$ on $T$. As one continues $T$ from $M_{1}$ through $P$, the arc $\dot{A}$ must either go to the origin or go off to infinity. In the latter case, the arc would have to intersect $M_{1}$ a second time, and $f[x(\tau)]$ would attain on $T$ either a relative maximum or a relative minimum value at a point $x=\xi \notin M_{1}$; that is, at an interior point of a subarc of $T$ within $M_{1}$. At $x=\xi$, we would then have

$$
f_{x_{1}} \frac{d x_{1}}{d \tau}+f_{x_{2}} \frac{d x_{2}}{d \tau}+\cdots+f_{x_{n}} \frac{d x_{n}}{d \tau}=0 .
$$

But since $x=\xi$ lies on $T$, equations (2) must be satisfied, and it follows that

$$
f_{x_{1}}^{2}+f_{x_{2}}^{2}+\cdots+f_{x_{n}}^{2}=0
$$


at $x=\xi$; that is, $x=\xi$ is a critical point of $f(x)$, contrary to hypothesis. Accordingly, the are $A$ that starts at $M_{1}$ and passes through $P$ goes to the origin. If $f(P) \geqq c_{1}$, it would follow that $f[x(\tau)]$ would possess an extremum at an interior point of $A$, and the argument employed above would show that this extremum would actually be a critical point of $f$. From this contradiction we infer that $f(P)<c_{1}$.

Suppose now that $P_{1}$ is any point of $E^{n} \notin D_{1}+M_{1}$. We shall show that $f\left(P_{1}\right)>c_{1}$. For, suppose $f\left(P_{1}\right) \leqq c_{1}$. Then we continue the trajectory $T_{1}$ through $P_{1}$ orthogonal to $M_{1}$ from $P_{1}$ to the origin. On this arc there would again be an extremum of the function $f[x(\tau)]$ that can be shown, as above, to be a critical point of $f$.

We combine the foregoing results in the following statement.

THEOREM. Let $f(x)$ be admissible and have no critical point, finite or infinite, except the origin. Then, $f(x)>0,(x) \neq(0)$, throughout $E^{n}-\{0\}$. The set of points $f(x)=c$, where $c$ is any (positive) value assumed by $f$, is a bounded closed manifold $M$ that bounds an (open) domain $D$ containing the origin. Further, $f(x)<c$ throughout $D$ and $f(x)>c$ exterior to $M$. Finally, if $0<c_{1}<c$, the closed manifold $f=c_{1}$ lies wholly in $D$.

The following corollary ${ }^{2}$ is an immediate consequence of the theorem.

COROLlary 1. If $f(x)$ is admissible and if $f\left(x_{0}\right) \leqq 0$ for some point $\left(x_{0}\right) \neq(0), f(x)$ has a critical point, finite or infinite, in addition to that at the origin.

We continue with a definition. A solution curve of (2) joining the origin to a point $P$ on which the only critical point of $f$ is the origin will be called an $\alpha$-arc joining these two points. We have then the following result.

CoRollaRY 2. If $f(x)$ is admissible and $f\left(x_{0}\right) \leqq 0,\left(x_{0}\right) \neq(0)$, there can be no $\alpha$-arc joining the origin to the point $(x)=\left(x_{0}\right)$.

For, the assumption of the existence of such an arc would lead, as above, to the existence of a critical point of $f$ on the arc.

The following examples will illuminate the theory.

EXAmPLE. The function $f(x, y)=y^{2}+x^{4}$ has precisely one critical point, the (degenerate) relative minimum point at the origin. The

2 The question answered by this corollary was put to the writer by Professor George Szegö. 
level lines $y^{2}+x^{4}=c(0<c<\infty)$ are closed ovals about the origin, and their orthogonal trajectories are the curves $x=0$ and

$$
y=k \exp \left(-1 / 4 x^{2}\right),
$$

$k$ constant. The trajectory through each point in the plane, except the origin, is clearly an $\alpha$-arc.

ExAmPle. Let $f$ be the function

$$
f(x, y)=6 x^{2}+y^{2}+2 x^{3} .
$$

Clearly, $f$ is positive definite at the origin and vanishes along a curve that passes through the point $(-5,10)$. Accordingly, $f$ must possess a critical point in addition to that at $(0,0)$. It is readily seen that this is the point $(-2,0)$. The equations $f(x, y)=c(0<c \leqq 8)$ determine closed curves around the origin. The trajectories orthogonal to these level lines are the curves

$$
y^{6}=k \frac{x}{x+2} .
$$

It will be observed, for example, that the trajectories orthogonal to the level curves at points $\left(x_{0}, y_{0}\right)$ for which $-2<x_{0}<0, y_{0} \neq 0$, start at the origin and go off to infinity asymptotic to the line $x=-2$, the abscissa of the second critical point. On the other hand, the line $y=0$ joins every point $P_{0}\left(x_{0}, 0\right), x_{0}<-2$, to the origin and is the trajectory through $P_{0}$ orthogonal to the given level lines. It clearly passes through the critical point $(-2,0)$. There is clearly no $\alpha$-arc passing through any point to the left of the line $x=-2$. All points except the origin for which $f \leqq 0$ lie to the left of this line.

Some of the preceding analysis can be recast as follows. Let $f(x)$ be a function of class $C^{\prime \prime}$ in $E^{n}$ with a relative minimum point at $x=a$, and suppose that $x=a$ is an isolated critical point of $f(x)$. If $f(a)=k$, the equation $f(x)=k+\varepsilon$, where $\varepsilon$ is a sufficiently small positive number, represents an $(n-1)$-manifold $M$ in a neighborhood of $x=a$. Through each point of $M$ there exists a unique trajectory orthogonal to $M$. We extend each such trajectory in both directions from $M$ terminating the extension only when we reach a critical point of $f$. Let $k$ be the point set union of all such trajectories with all critical points deleted. Finally, let $B$ be set of all points in $E^{n}$ for which $f(x) \leqq k$. It follows that $K \cap B=\varnothing$.

An analogous result may, of course, be stated when $x=a$ is a relative maximum point of $f$. 


\section{BIBLIOGRAPHY}

1. G.A. Bliss, Fundamental existence theorems, American Mathematical Society, New York, 1913, p. 95.

2. Walter Leighton, Morse theory and Liapunov functions, Rend. del Circolo Matematico, Serie II (1964), 1-10.

3. Marston Morse, Relations between the critical points of a real function of $n$ independent variables, Trans. Amer. Math. Soc. 27 (1925), 345-396.

Received March 11, 1965. This will acknowledge the partial support of the author by the U.S. Army Research Office (Durham) under Grant numbered DAARO(D)-31-124-G-600. Reproduction in whole or in part is permitted for any purpose of the United States Government.

WESTERN RESERVE UNIVERSITY 


\section{PACIFIC JOURNAL OF MATHEMATICS}

\section{EDITORS}

H. SAMELSON

Stanford University

Stanford, California

J. P. JANS

University of Washington

Seattle, Washington 98105

\section{J. DuGUNDJI}

University of Southern California

Los Angeles, California 90007

RICHARD ARENS

University of California

Los Angeles, California 90024

\section{ASSOCIATE EDITORS}

E. F. BECKENBACH
B. H. NEUMANN

F. WOLF

K. YoSIDA

\section{SUPPORTING INSTITUTIONS}

\author{
UNIVERSITY OF BRITISH COLUMBIA \\ CALIFORNIA INSTITUTE OF TECHNOLOGY \\ UNIVERSITY OF CALIFORNIA \\ MONTANA STATE UNIVERSITY \\ UNIVERSITY OF NEVADA \\ NEW MEXICO STATE UNIVERSITY \\ OREGON STATE UNIVERSITY \\ UNIVERSITY OF OREGON \\ OSAKA UNIVERSITY \\ UNIVERSITY OF SOUTHERN CALIFORNIA
}

\author{
STANFORD UNIVERSITY \\ UNIVERSITY OF TOKYO \\ UNIVERSITY OF UTAH \\ WASHINGTON STATE UNIVERSITY \\ UNIVERSITY OF WASHINGTON \\ AMERICAN MATHEMATICAL SOCIETY \\ CHEVRON RESEARCH CORPORATION \\ TRW SYSTEMS \\ NAVAL ORDNANCE TEST STATION
}

Mathematical papers intended for publication in the Pacific Journal of Mathematics should be typewritten (double spaced). The first paragraph or two must be capable of being used separately as a synopsis of the entire paper. It should not contain references to the bibliography. Manuscripts may be sent to any one of the four editors. All other communications to the editors should be addressed to the managing editor, Richard Arens at the University of California, Los Angeles, California 90024 .

50 reprints per author of each article are furnished free of charge; additional copies may be obtained at cost in multiples of 50 .

The Pacific Journal of Mathematics is published monthly. Effective with Volume 16 the price per volume (3 numbers) is $\$ 8.00$; single issues, $\$ 3.00$. Special price for current issues to individual faculty members of supporting institutions and to individual members of the American Mathematical Society: $\$ 4.00$ per volume; single issues $\$ 1.50$. Back numbers are available.

Subscriptions, orders for back numbers, and changes of address should be sent to Pacific Journal of Mathematics, 103 Highland Boulevard, Berkeley 8, California.

Printed at Kokusai Bunken Insatsusha (International Academic Printing Co., Ltd.), No. 6, 2-chome, Fujimi-cho, Chiyoda-ku, Tokyo, Japan.

\section{PUBLISHED BY PACIFIC JOURNAL OF MATHEMATICS, A NON-PROFIT CORPORATION}

The Supporting Institutions listed above contribute to the cost of publication of this Journal, but they are not owners or publishers and have no responsibility for its content or policies. 


\section{Pacific Journal of Mathematics

Vol. 19, No. $3 \quad$ July, 1966

S. J. Bernau, The spectral theorem for unbounded normal operators .......

Lu-san Chen, Asymptotic behavior of solutions of parabolic equations of

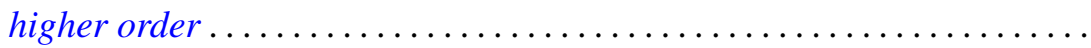

Lawrence William Conlon, An application of the Bott suspension map to the

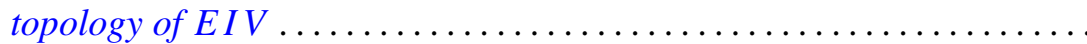

Neal Eugene Foland and John M. Marr, Sets with zero-dimensional kernels .........................................

Stanley Phillip Franklin and R. H. Sorgenfrey, Closed and image-closed

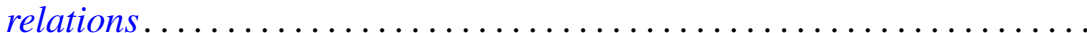

William Jesse Gray, A note on topological transformation groups with a

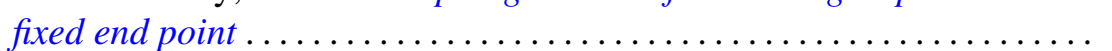

Myron Goldstein, $K$ - and L-kernels on an arbitrary Riemann surface ...... 449

George Joseph Kertz and Francis Regan, The exponential analogue of a generalized Weierstrass series .............................

Walter Leighton, On Liapunov functions with a single critical point ........ 467

Bernard Werner Levinger and Richard Steven Varga, On a problem of $O$.

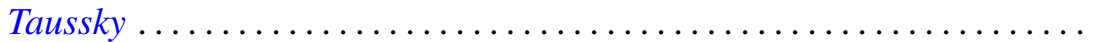

Lowell Duane Loveland, Tame subsets of spheres in $E^{3} \ldots \ldots \ldots \ldots \ldots . .489$

Erik Andrew Schreiner, Modular pairs in orthomodular lattices ......... 519

K. N. Srivastava, On dual series relations involving Laguerre polynomials ...............................

Arthur Steger, Diagonability of idempotent matrices.....

Walter Strauss, On continuity of functions with values in various Banach spaces...

Robert Vermes, On the zeros of a linear combination of polynomials ...

Elliot Carl Weinberg, On the scarcity of lattice-ordered matrix rings ....

Harold Widom, Toeplitz operators on $H_{p} \ldots \ldots \ldots \ldots \ldots$

Neal Zierler, On the lattice of closed subspaces of Hilbert space...

Irving Leonard Glicksberg, Correction to: "Maximal algebras and a theorem of Rado"

John Spurgeon Bradley, Correction to: "Adjoint quasi-differential operators of Euler type"

William Branham Jones, Erratum: "Duality and types of completeness in locally covex spaces".

Stanley P. Gudder, Erratum: "Uniqueness and existence properties of bounded observables" 